\title{
Intraoperative salvage endoscopy performed during orthotopic liver transplantation due to esophageal bleeding
}

\author{
Konrad Kobryn, Sławomir Kozieł, Waldemar Patkowski, Michał Grąt, Tadeusz Wróblewski, Marek Krawczyk \\ Department of General, Transplant and Liver Surgery, Medical University of Warsaw, Warsaw, Poland
}

Videosurgery Miniinv 2015; 10 (3): 472-476

DOI: $10.5114 /$ wiitm.2015.54559

\begin{abstract}
Introduction: Liver transplantation (LTX) is a widely accepted method of treatment for end stage liver diseases. There are many reports on the management of gastrointestinal bleeding (GIB) after $L T x$, however the number of studies concerning salvage endoscopic procedures during LTX are scarce.

Aim: We present our material of intraoperative endoscopic procedures due to GIB during LTX.

Material and methods: During this period there were 4 females and 1 male at the mean age of 52.2 (35-65) years who underwent LTX and 1 patient had Re-LTX. All patients were Child-Pugh group C and mean MELD score was 17.75. Esophageal and/or gastric varices were present before surgery in all patients but only 1 female patient didn't experience GIB prior to LTX. Variables such as operating time, cold ischemic time, blood loss, blood transfusion, PLT count, international normalized ratio, albumin levels were similar in all patients thus making it statistically insignificant as the cause of $G I B$.

Results: In all cases a single IOE was necessary and bleeding from ruptured varices succumbed to endoscopic ligation. In 2 patients besides trials of ligating the varix, histoacryl was put in use which proved success. In both these last female patients the endoscopic physician had to insert a Danis stent. A follow up endoscopy was performed on the 7-10 POD.

Conclusions: Intra-operative endoscopy performed during LTX does not interrupt surgery. Performed as soon as possible results in less future endoscopic interventions due to GIB. Intraoperative endoscopy may be considered as a salvage procedure and should be performed in the shortest possible time.
\end{abstract}

Key words: liver transplantation, endoscopy, gastrointestinal bleeding, portal hypertensive gastropathy.

\section{Introduction}

Liver transplantation (LTX) is a widely accepted and the only reasonable method of treatment for end stage liver disease. To this day it is performed in many transplant centers worldwide. With the understanding of immunosuppression, attained knowledge and surgical experience, and improvement of peri-operative treatment, there is a continual expansion of indications for LTX, as well as better management of intra- and post-operative complications [1-4]. There are many reports on the treatment and management of gastrointestinal bleeding (GIB) after LTx, but studies concerning salvage endoscopic procedures during liver graft explantation or implantation are scarce.

Our 24-year liver transplant program, initiated in 1991, allowed the department to establish an oncall multidisciplinary team consisting of not only surgeons but also intensive care physicians, hepatologists and endoscopic physicians. This resulted in reducing risk factors of liver transplant patients and extending general post-LTx survival. With over

\section{Address for correspondence}

Konrad Kobryn MD, Department of General, Transplant and Liver Surgery, Medical University of Warsaw, 1 A Banacha St, 02-097 Warsaw, Poland, phone: +48 606736 505, e-mail: konradkobryn@gmail.com 
1500 LTx performed, we analyzed 521 patients who underwent LTx between 2012 and 2014. There were 5 salvage intra-operative endoscopic (IOE) procedures conducted during abdominal surgery in $5(0.96 \%)$ recipients in this period due to gastrointestinal bleeding (GIB).

Gastrointestinal bleeding is most commonly caused by ruptured varix (RV), portal hypertensive gastropathy (PHG), peptic ulcers (PU), bleeding from jejunojejunostomy anastomosis and ulceration [4-8]. End stage liver failure often coexists with portal hypertension, which is expressed in splenic hypertrophy, esophageal varices and the above-mentioned portal hypertensive gastropathy $[2,3]$. Portal hypertensive gastropathy is the change in the gastric mucosa of the patient with portal hypertension, and mucosal changes of PHG can be defined as the presence of mucosal friability and dilated blood vessels in the mucosal surface [9].

Patients awaiting liver transplantation routinely undergo endoscopic checkup and therapy. This is intensified after the first incidence of GIB. The procedure of endoscopic treatment escalates from clipping to banding to variceal argon beamer ligation and intensifies to somatostatin and adrenaline, vasopressin injections, resulting in stent implantation due to excessive and difficult to manage bleeding [7, 10-13]. The use of oesophageal stents is well known in other diseases of the esophagus as a tumor stenosis or perforation and mediastinitis. The recognized risk factors for gastrointestinal bleeding are shown in Table I. However, GIB is more frequent with primary graft dysfunction (PGD) than without.

Gastrointestinal bleeding is considered life threatening. Mortality rates found in the literature range from $4 \%$ to $15 \%$ [7-9]. However, concerning cirrhotic patients these statistics are higher and result in higher mortality and morbidity. The most common cause of GIB in cirrhotic liver patients is esophageal and gastric variceal bleeding (up to $60 \%$ ). Other causes of GIB which represent the remaining $40 \%$ are portal hypertensive gastropathy, peptic ulcers, malignancy, angiodysplasia, and bleeding from jejunojejunostomy anastomosis, and the treatment of them can be varied and usually is very complex [11, 12].

\section{Material and methods}

During this period there were 4 females and 1 male at the mean age of $52.2(35-65)$ years among the 5 patients who had IOE due to GIB. Two females with HCV-positive cirrhosis and chronic liver failure underwent elective liver transplantation. The third female patient, treated for Wilson disease (WD), received an urgent liver transplant due to acute liver failure, whilst the fourth female received a liver transplant due to end stage liver failure with an etiology of alcoholic liver disease (ALD) and concomitant factors of Wilson disease. The remaining male was also diagnosed with ALD but co-existing $\mathrm{HCV}$ cirrhosis, and he underwent an urgent liver retransplantation (Re-LTX) due to primary non-function (PNF) of the graft implanted $48 \mathrm{~h}$ before. All patients were classified in Child group $C$. The mean MELD score in the group was 17.75 (range: 14-24). Esophageal and/or gastric varices were present before surgery in all patients but only 1 female patient with WD did not experience gastrointestinal bleeding prior to LTx. All liver transplantations were performed using the piggy-back technique, while the Re-LTx was conducted according to the classical technique using a veno-venous bypass. Blood retrieval through the cell-saver was not used. Factors during surgery, such as operating time, cold ischemic time, blood loss, blood transfusion, platelet count, international normalized ratio (INR), and albumin levels were similar in all patients, thus making it statistically insignificant as the cause of gastrointestinal bleeding (Table II).

The scenario in all cases is basically the same. Intra-operatively the surgeons notice the stomach filling up, becoming bulky and steadily starting to interfere with access towards the hepatoduodenal ligament and its further preparation. The anesthesiologist is asked to deflate the stomach with the gastro-nasal tube. While doing so, he retrieves and collects blood fluid into the suction tank. Now, apart from this being another risk factor for the patient, it does not prevent the surgeon from continuing to operate, but it definitely impairs maneuvers around the hepato-duodenal ligament.

Table I. Risk factors for gastrointestinal bleeding

\begin{tabular}{|c|}
\hline Intra-operative blood loss $>81$ \\
\hline Hepaticojejunostomy \\
\hline Portal vein pressure $>20 \mathrm{~mm} \mathrm{Hg}$ \\
\hline Bilirubin level $>20 \mathrm{mg} /$ dl post LTx (PGD: primary graft \\
dysfunction)
\end{tabular}


Table II. Patient characteristics

\begin{tabular}{|lcccccc|}
\hline Patient & Age [years] & MELD Score & GIB pre-LTx & HCV & WD & ALD \\
\hline Female & 65 & 18 & + & + & & \\
\hline Female & 50 & 15 & + & & + & + \\
\hline Female & 45 & 14 & + & & + \\
\hline Female & 35 & 15 & + & & + \\
\hline Male & 66 & 24 & & & & + \\
\hline
\end{tabular}

\section{Results}

In our study 2 patients (P1 with WD and P2 with $\mathrm{HCV}+$ cirrhosis) were attended during LTx by a laryngologist on call and received a frontal tamponade of the nose prior to endoscopy. Out of the $5 \mathrm{IOE}$ procedures, 2 were performed during hepatectomy (the removal of the diseased liver), 1 IOE took place during warm ischemia (the period during which the

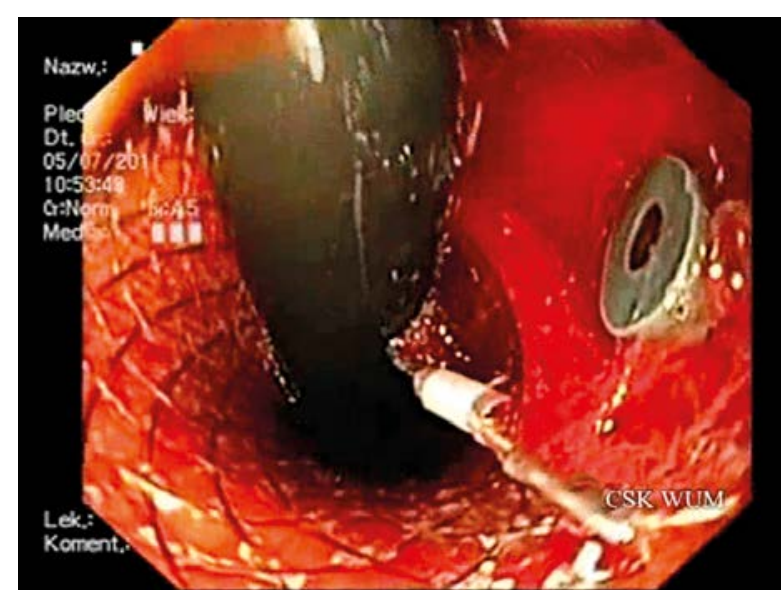

Photo 1. Danis stent antrum view

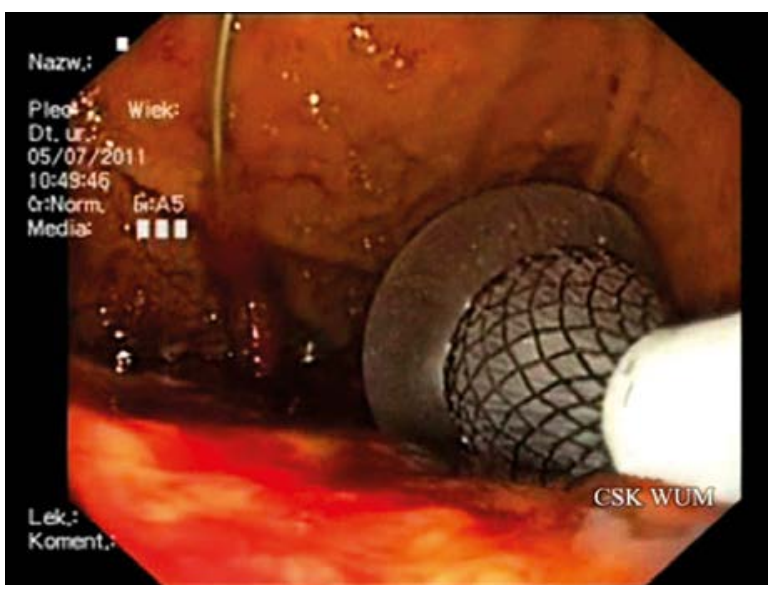

Photo 2. Danis stent placement diseased liver is explanted, but the recovered graft is not yet implanted - during this time the surgeons usually attend to adequate hemostasis in the abdominal cavity), in the $4^{\text {th }}$ case GIB occurred at the beginning of implantation and the IOE was performed before reperfusion of the graft, while in the $5^{\text {th }}$ patient fluid restraints and close to zero intraoperative blood loss allowed the operation to be continued, keeping the patient hemodynamically stable; thus IOE was performed during abdominal closure.

In all cases a single IOE was necessary, and bleeding from ruptured varices succumbed to endoscopic ligation. In two patients besides trials of ligating the varix, Histoacryl was put in use, which proved successful in the patient with Wilson disease. Unfortunately, the above methods were not satisfactory in 2 female patients -1 with HCV+ cirrhosis alone, who as we learned post-operatively had 3 incidents of bleeding from esophageal varices prior to the transplantation, and the other with HCV and concomitant ALD. In both these female patients the endoscopic physician had to insert a stent (SX-ELLA Stent Danis), as shown in Photos 1-3.

In all cases during endoscopy the surgeons continued with the operation. All patients lived through

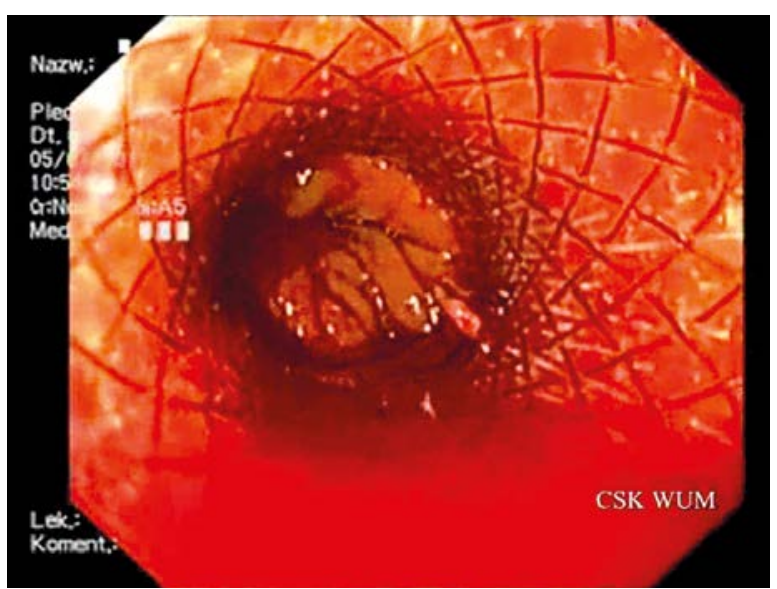

Photo 3. Danis stent insertion in GIB 
surgery and were managed in an intensive care unit. No excessive bleeding from the gastrointestinal tract was observed, neither directly post-LTx, nor during the next 4-5 postoperative days (POD). The patients with the Danis stent had it removed on the $10^{\text {th }}$ and $8^{\text {th }}$ POD without any complications. The first female patient with the Danis stent after LTx was discharged from hospital in the $3^{\text {rd }}$ post-operative week in good general condition and is now, 2 years after LTx, under routine control in the outpatient clinic, while the second one was referred to the Hepatology Department in good condition with improving lab results.

We did not observe any further incidents of GIB in the male patient after the Re-LTX. With satisfactory results of the new liver graft, he however never regained proper kidney function, had recurring systemic infections and died from multi-organ failure 44 days after Re-LTX.

No complications were observed in the second $\mathrm{HCV}+$ female recipient, so no control endoscopy was intended. She was discharged in very good general condition on the $16^{\text {th }}$ POD. One year after liver transplantation she was examined in the outpatient clinic with good results.

The female patient with Wilson disease who underwent an urgent LTx recovered well and was discharged without any signs of GIB. She was seen 7 weeks after LTx in the outpatient clinic because of jaundice. She was admitted to hospital the same day and scheduled for endoscopic retrograde cholangiopancreatography, this being the only reason for an endoscopic procedure. She had an end-to-end bile duct anastomosis stricture which was expanded and a plastic stent was applied to the right hepatic duct. Treatment was successful, and the patient was discharged from the Surgery Department. She was admitted a couple weeks later due to graft rejection (ABO incompatibility complex) and underwent a liver re-transplantation. The peri-operative period passed without complications. She was discharged in good condition. Currently 14 months after Re-LTx she remains in good general condition with satisfactory graft function.

\section{Discussion}

Liver transplantation is the only method of treatment for end stage liver disease. With a properly executed LTx qualification protocol, patients undergo a better selection in order to receive a transplant in the best possible period of end stage disease. How- ever, GIB due to portal hypertension tends to occur more frequently in certain patients, i.e. more often than once every 6-8 weeks [11-13]. With stable hepatic lab results (AST, ALT, TBil, etc.), gastrointestinal bleeding from esophageal varices tends to be the worst scenario for such patients. In this group, patients called in for liver transplantation are routinely examined. The blood morphology, serum test, coagulogram, chest X-ray, ECG, abdominal USG and sometimes abdominal computed tomography (CT) are performed before LTX, but the qualifying physician has no knowledge of whether or not esophageal varices are enlarged and advanced in degree as in comparison with the last gastroscopy. So the transplant team receives a well-prepared LTx recipient in the operating theater but with no practical information on the variceal stage and risk of bleeding. During intubation the anesthesiologist does not notice any impairment from the esophagus; however, during the implementation of the gastric tube the first signs of bleeding may appear (POINT 1). When the surgeon asks to reposition the gastric tube, it is the second time when manual irritation is applied to the esophageal wall (POINT 2). After that, if the varices are at any risk of bleeding, it will not take long for the anesthesiologist to notice the collection of blood in the gastric tube. At this point usually the transplant surgeons are dissecting the hepatoduodenal ligament, preparing for ligation of the common bile duct and hepatic artery. This is usually the time to call the unit's on-call endoscopist.

In our opinion, it is crucial for a large transplant unit to have among its staff a physician who performs endoscopy before and after liver transplant, as the volume of these procedures is so high that the experience in GIB management is extensive, especially among PHG patients. Our center has two highly experienced endoscopic surgeons, one of whom is always on-call. Time from paging the endoscopist to the beginning of the endoscopic procedure is $<60 \mathrm{~min}$.

As mentioned above in the paper, all patients who during surgery were treated by IOE had all ruptured varices obliterated successfully. The challenge is that the endoscopy takes place in the operating room, where there are already plenty of devices and little free space. The anesthesiologist needs a free approach to all the IVs and anesthetic column - this provides little space for the performing endoscopic surgeon to maneuver the endoscope. Another issue of concern is that the patient is sedated and stabi- 
lized for LTx, so he cannot be moved around in order to achieve the right angle in endoscopy. The only difference is that where necessary the transplant surgeon may maneuver the stomach during laparotomy in consultation with the endoscopic physician. Situations such as intraoperative gastrointestinal bleeding are demanding for the anesthesiologists because every unit of RBC and plasma they admit is a certain nuisance to the LT surgeons, who have to carry out their operation with as little blood loss as possible.

In our experience, usually the endoscopic procedure can be performed without interrupting the liver transplantation. The duration of the procedure conducted by an experienced endoscopist does not exceed one hour in order to stop the bleeding. In most cases if management of IO GIB fails with the use of an argon beamer or cyanoacrylate sclerotherapy, a DANIS stent should be applied in order to briefly halt bleeding and reduce blood loss during LTx $[12,13]$. All patients who have IOE have to undergo endoscopic follow-up in the first few days after transplantation. We suggest performing the first endoscopic follow-up during POD 6-8 in order to remove the stent under safe conditions and stable coagulogram results.

\section{Conclusions}

Intra-operative endoscopy performed during liver transplantation does not interrupt surgery. Performing it as soon as possible results in fewer future endoscopic interventions due to gastrointestinal bleeding. Any complications resulting from endoscopy may be attended to during open surgery. Since liver transplantation is an operation with increased risk of blood loss, excessive blood loss to the gastrointestinal tract is unnecessary and reduces survival; thus IOE may be considered as a salvage procedure and should be performed in the shortest possible time.

\section{Conflict of interest}

The authors declare no conflict of interest.

\section{References}

1. Tabasco-Minguillan J, Jain A, Naik M, et al. Gastrointestinal bleeding after liver transplantation. Transplantation 1997; 63: 60-7.

2. Hashizume M, Tanaka K, Inokuchi K. Morphology of gastric microcirculation in cirrhosis. Hepatology 1983; 3: 1008-12.
3. Nyckowski P, Pawlak J, Zieniewicz K, et al. Liver transplantation in patients with hepatic failure. Transplant Proc 2002; 34: 631-4.

4. Hirata M, Kita Y, Harihara Y, et al. Gastrointestinal bleeding after living-related liver transplantation. Dig Dis Sci 2002; 47: 2386-8.

5. Icoz G, Kilic M, Zeytunlu M, et al. Roux-en-Y bleeding after living donor liver transplantation: a novel technique for surgical treatment. Transplant Proc 2003; 35: 1463-5.

6. Kimura K, Ikegami T, Bekki Y, et al. Clinical significance of gastrointestinal bleeding after living donor liver transplantation. Transplant Int 2014; 27: 705-11.

7. Lau JY, Barkun A, Fan DM, et al. Challenges in the management of acute peptic ulcer bleeding. Lancet 2013; 381: 2033-43.

8. Hearnshaw SA, Logan RF, Lowe D, et al. Acute upper gastrointestinal bleeding in the UK: patient characteristics, diagnoses and outcomes in the 2007 UK audit. Gut 2011; 60: 1327-35.

9. Ripoll C, Garcia-Tsao G. Management of gastropathy and gastric ectasia in portal hypertension. Clin Liver Dis 2010; 14: 281-95.

10. Kovacs TO, Jensen DM. Endoscopic treatment of ulcer bleeding. Curr Treat Options Gastroenterol 2007; 10: 143-8.

11. Lau JY, Sung JJ, Lam YH, et al. Endoscopic retreatment compared with surgery in patients with recurrent bleeding after initial endoscopic control of bleeding ulcers. N Engl J Med 1999; 340: 751-6.

12. Cameron R, Binmoeller KF. Cyanoacrylate applications in the Gl tract. Gastrointest Endosc 2013; 77: 846-57.

13. Cheng LF, Wang ZQ, Li CZ, et al. Treatment of gastric varices by endoscopic sclerotherapy using butyl cyanoacrylate: 10 years' experience of 635 cases. Chin Med J (Engl) 2007; 120: 2081-5.

Received: 23.06.2015, accepted: 17.09.2015. 